Bolm Inst. oceanogr. S Paulo, 40(1/2):15-25, 1992

\title{
Migraciones verticales de Alexandrium excavatum (Braarud) Balech et Tangen en columnas experimentales*
}

\author{
Betina Andrea SANTOS ** \& Jose Ignacio CARRETO
}

Instituto Nacional de Investigación y Desarrollo Pesquero

(Casilla de Correo 175, 7600 Mar del Plata, Argentina)

\begin{abstract}
- Abstract: Diel vertical migrations of the marine dinoflagellate $A$. excavatum were followed in a laboratory water column, and the effects of temperature stratification and nitrogen depletion on patterns of migration were examined. It was found that A. excavatum is a vertical migrator aggregating at surface during daylight, and descending at night. A six-degree thermocline did not restrict its migration but delayed the vertical movements. Nitrogen depletion in the culture was correlated with earlier downward migration in the day, and nitrate assimilation in the dark was confirmed. In another essay, with a nitrogen deficient culture, nocturnal nitrate uptake per cell was measured. It is suggested that the ability of $A$. excavatum to take up nitrate in the dark working together with diurnal vertical migration could be important mechanisms regulating bloom developments.
\end{abstract}

- Descriptors: Vertical migrations, Bioassays, Phytoplankton culture, Nyctimeral rhythms, Light effects, Temperature effects, Nutrients (mineral), Dinoflagellata, Alexandrium excavatum.

- Descritores: Migrações verticais, Bioensaios, Cultivo de fitoplâncton, Rítmos nictemerais, Efeitos da luz, Efeitos da temperatura, Nutrientes (mineral), Dinoflagellata, Alexandrium excavatum.

\section{Introducción}

Las mareas rojas tóxicas registradas en el Mar Argentino desde noviembre de 1980, han sido causadas por el dinoflagelado Alexandrium excavatum (Braarud) Balech et Tangen (Carreto et al., 1981). El desarrollo de estos eventos estuvo asociado a frentes hidrográficos bien delimitados, caracterizados por el encuentro de aguas verticalmente homogéneas con otras bien estratificadas (Carreto et al., 1981; 1985; 1986). En estos sistemas la concentración de nutrientes presenta marcadas diferencias. En las aguas verticalmente uniformes las concentraciones de nitratos son mayores que las correspondientes a la capa superficial de la zona estratificada aunque mucho menores que las halladas en ésta última por debajo de la termoclina. En las aguas

(*) Contribución Ne 824 del Instituto Nacional de Investigación y Desarrollo Pesquero, Argentina

(*) Becaria de la Universidad Nacional de Mar del Plata. homogéneas predominan las diatomeas y en las estratificadas los dinoflagelados encontrándose las mayores concentraciones de $A$. excavatum en la zona frontal.

Por su motilidad y fototaxismo los dinoflagelados son capaces de realizar migraciones verticales através de la columna de agua. Esta capacidad constituye una ventaja adaptativa sobre los organismos inmóviles tales como las diatomeas en aguas estratificadas, ya que les permitirfa explotar los nutrientes existentes por debajo de la termoclina durante la noche, asimilando anhídrido carbónico en las capas superficiales bien iluminadas durante el día (Eppley \& Harrison, 1975; Harrison, 1976). Se ha señalado sin embargo, que no todas las especies de dinoflagelados siguen este patrón de migración. Por otra parte, el comportamiento de una determinada especie presenta notables alteraciones, de acuerdo al estado fisiológico de la misma y las condiciones del ambiente en que se desarrolla (Forward, 1976 y referencias allí citadas).

En el presente trabajo se estudia el comportamiento migratorio que sigue $A$. excavatum en columnas 
experimentales a escala reducida que simulan condiciones ambientales observadas en la naturaleza y se ensaya la capacidad de esta especie para asimilar nitratos en la oscuridad. Finalmente se discuten los resultados experimentales obtenidos con la distribución de esta especie observada durante el desarrollo de una marea roja.

\section{Materiales y métodos}

El cultivo de $A$. excavatum se obtuvo de la colección del Laboratorio de Química del Agua de Mar y Bioproducción del INIDEP. El mismo era mantenido en medio f/2 (Guillard \& Ryther, 1962), con iluminación cíclica de 14 $\mathrm{L}: 10 \mathrm{O}, 4000$ lux de intensidad y $18^{\circ} \mathrm{C}$ de temperatura, en una cámara de cultivos.

\section{Experiencia $n^{2} 1$}

Se utilizó una columna de vidrio de $50 \mathrm{~cm}$ de altura y 8 $\mathrm{cm}$ de diámetro a la que se le agregó medio $\mathrm{f} / 2$ y el cultivo de $A$. excavatum hasta alcanzar un volumen de 21 . Se colocó esta columna en la cámara de cultivos, iniciando el período luminoso a las $9 \mathrm{~h}$ y finalizando a las $23 \mathrm{~h}$. Se cubrieron casi las $3 / 4$ partes inferiores de manera de oscurecer su interior. El cuarto superior recibió iluminación lateral de una lámpara de tungsteno, siendo la intensidad sobre la zona superficial de 8350 lux y 2500 lux en la zona de transición luz/oscuridad (Fig. 1a).

La temperatura del medio al comenzar el período de luz en superficie era de $18.7^{\circ} \mathrm{C}, 17^{\circ} \mathrm{C}$ en la transición y $16.8^{\circ} \mathrm{C}$ en el fondo, ascendiendo durante el día (debido al calor proporcionado por la lámpara) a $24^{\circ} \mathrm{C}$ en superficie, $20^{\circ} \mathrm{C}$ en la transición y $19.6^{\circ} \mathrm{C}$ en el fondo. En el transcurso de un día, se extrajeron muestras para conocer el $\mathrm{n}^{2} \mathrm{cel}^{\mathrm{ml}}{ }^{-1}$, en tres niveles de la columna: superior, transición e inferior. Para esto se utilizaron tubos de vidrio con el extremo inferior terminado en capilar cerrado. Estos tubos se sumergían hasta las profundidades deseadas y por presión contra la pared de la columna se producía la ruptura de sus extremos, lo que permitía aspirar la muestra de cada una de las profundidades seleccionadas sin efectos de arrastre.

\section{Experiencia $\mathbf{n}^{0} 2$}

La columna de cultivo utilizada en la experiencia anterior se colocó en un recipiente de vidrio separado en dos compartimientos estancos por un tabique central. En la sección inferior circulaba agua que era enfriada al pasar por un baño térmico hasta $15^{\circ} \mathrm{C}$. En la zona superior se mantenía otro baño a $21 \pm 1^{\circ} \mathrm{C}$, con un calefactor termostatizado. La iluminación era proveniente de dos tubos fluorescentes (luz/día), conectados a un interruptor cíclico (14 L:10 O), que proporcionaba en la mitad superior una intensidad luminosa de 4000 lux. El período luminoso comenzaba a las $6 \mathrm{~h}$ y finalizaba a las $20 \mathrm{~h}$.

Las muestras para determinar la concentración celular, en los tres niveles durante el transcurso de un día, se obtuvieron mediante un muestreador fijo que consistía en tres tubos de vidrio introducidos en un tapón de la columna, cuyos extremos inferiores permanecían a las profundidades deseadas y los superiores estaban conectados a jeringas donde se colectaban las muestras por succión (Fig. 1b).

\section{Experiencia $\mathbf{n}^{0} 3$}

El sistema utilizado difiere de la experiencia anterior en que ejerce un mayor control sobre la temperatura al circular agua por la mitad superior de la caja de manera de establecer un gradiente constante de densidad dentro de la columna (Fig. $1 \mathrm{~b}$ ). La zona iluminada mantuvo una temperatura de $20^{\circ} \mathrm{C}$, decreciendo hasta $17.7^{\circ} \mathrm{C}$ en la transición, $16.5^{\circ} \mathrm{C}$ en la zona oscura, siendo en el fondo de $14.5^{\circ} \mathrm{C}$. El cultivo utilizado se desarrollo en medio $\mathrm{f} / 2$ ( $\sin$ el agregado de nitratos) durante dos semanas. Antes de hacer el inóculo se comprobó mediante observación microscópica que las células evidenciaban una característica de los cultivos deficientes en nitrógeno como es la pérdida de pigmentación. El cultivo se concentró, mediante filtración inversa, de manera que al agregar un pequeño volumen de éste no afectara la estabilidad de la columna. Dentro de ésta se colocó el mismo medio de cultivos ( $f / 2$ sin el agregado de nitratos). Las escasas concentraciones de este nutriente, provenientes del agua de mar utilizada en la preparación del medio, se distribuían en forma homogénea en la columna hasta que se agregó una solución concentrada de nitratos, previamente enfriada, por medio de un fino tubo de vidrio, en el fondo del recipiente. Se logró así la siguiente distribución:

$$
\begin{aligned}
& \text { nivel superior: } 0.61 \mu{\mathrm{M} \mathrm{N}-\mathrm{NO}_{3}-} \\
& \text { transicion } \quad: 0.65 \mu \mathrm{M} \mathrm{N}-\mathrm{NO}_{3}- \\
& \text { nivel inferior: } 6.11 \mu \mathrm{M} \mathrm{N}-\mathrm{NO}_{3}-
\end{aligned}
$$

Estos valores mostraban que la diferencia de densidad, que determinaba la estratificación de la columna, limitaba la difusión del nutriente hacia el nivel superior. Bajo estas condiciones se utilizó el mismo sistema descripto para la experiencia anterior. Cada 3 horas, durante una jornada, 


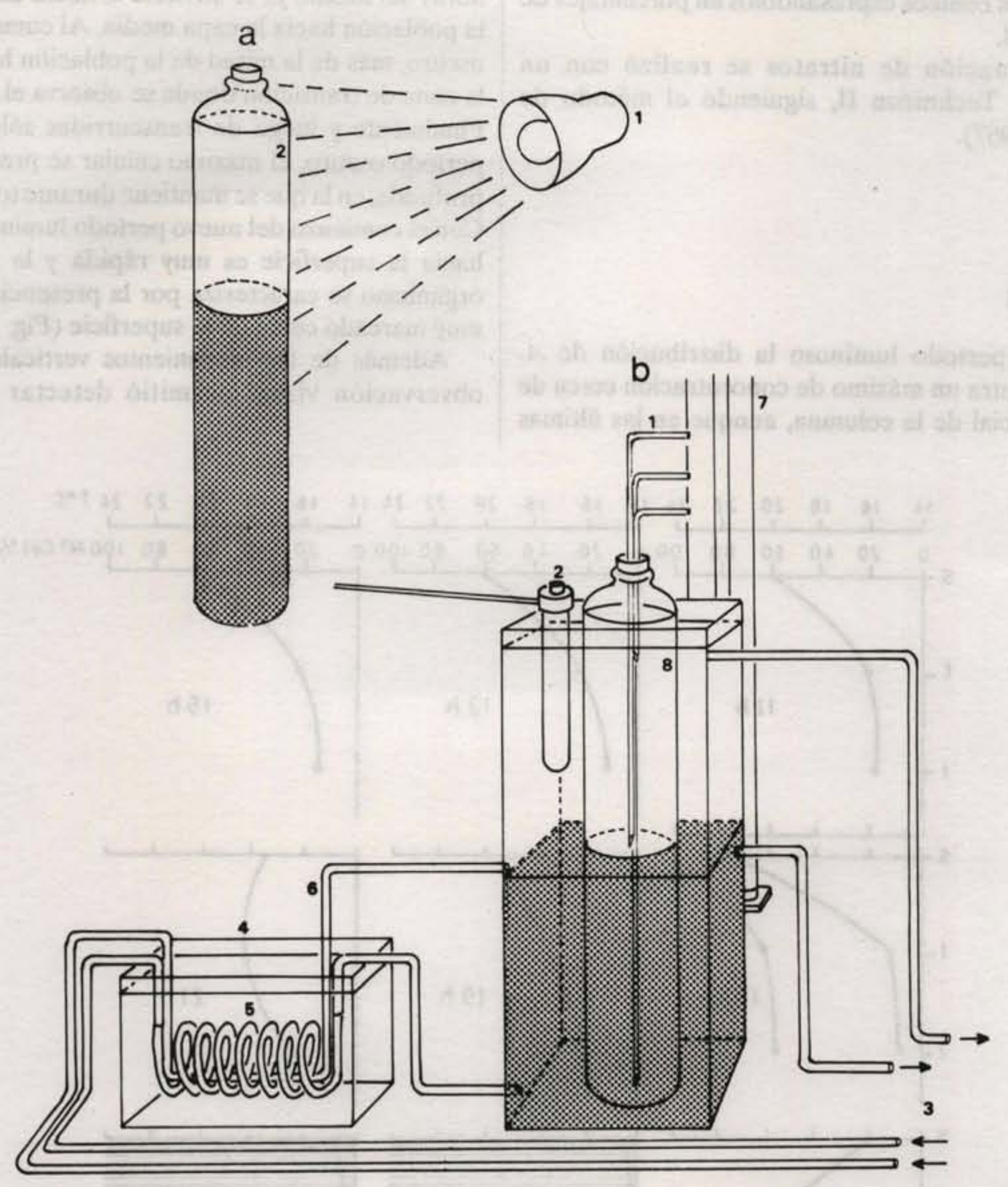

Fig. 1. a. Esquema del sistema utilizado en la experiencia $n \circ$ 1. 1: sistema de iluminación, 2: columna de cultivo. b. Esquema del sistema utilizado en las experiencias $n=2$ у 3. 1: sistema de muestreo, 2: calefactor termostatizado, 3: circulación de agua, 4: baño termostático, 5: serpentina de cobre, 6: tubo de circulación simple (incorporado en la 3era. experiencia, para la circulación de agua en la mitad superior de la caja), 7: sistema de lluminación, 8: columna de cultivo.

se tomaron muestras para determinar la concentración de nitratos y el $\mathrm{n}^{\circ} \mathrm{cel} . \mathrm{ml}^{-1}$ en cada nivel.

\section{Experiencia n 4}

En otro ensayo se desarrollo un cultivo de $A$. excavatum en un medio $f / 2$ sin el agregado de nitratos, en un recipiente de $200 \mathrm{ml}$, durante dos semanas, en la cámara a $18^{\circ} \mathrm{C}$ y con el ciclo $14 \mathrm{~L}: 10 \mathrm{O}$. Con el objeto de confirmar el consumo de nitratos en la oscuridad, se utilizó este cultivo cuando ya presentaba pérdida de pigmentación. Se agregó una solución concentrada de nitratos determinándose la concentración inicial de este nutriente en el medio (34.39 $\mu \mathrm{M} \mathrm{N}-\mathrm{NO}_{3}$, Fig. 5) y su velocidad de desaparición con una frecuencia de muestreo de 1 a 3 horas, considerando soblo el período oscuro. Además se determinó el $\mathrm{n}^{2} \mathrm{cel} \cdot \mathrm{ml}^{-1}$ al iniciar $(0 \mathrm{~h})$ y al finalizar el ensayo $(9 \mathrm{~h})$, lo que permitio calcular la tasa de incorporación de este nutriente por célula.

\section{Métodos analíticos}

La determinación del $\mathrm{n}^{\circ} \mathrm{cel} . \mathrm{ml}^{-1}$ se realizó en la primera experiencia en base al método de Fournier (1978) y en las restantes siguiendo la técnica de Utermöhl (1958). Se 
normalizaron los conteos expresándolos en porcentajes de células por nivel.

La determinación de nitratos se realizó con un autoanalizador Technicon II, siguiendo el método de Wood et al. (1967).

\section{Resultados}

\section{Experiencia $n^{01}$}

Durante el período luminoso la distribución de $A$. excavatum muestra un máximo de concentración cerca de la capa superficial de la columna, aunque en las últimas horas del mismo ya se advierte el inicio de un descenso de la población hacia la capa media. Al comenzar el período oscuro, más de la mitad de la población ha migrado hacia la zona de transición donde se observa el máximo celular. Finalmente y luego de transcurridas sólo dos horas del período oscuro, el máximo celular se presenta en la capa profunda, en la que se mantiene durante todo este período. Con el comienzo del nuevo período luminoso la migración hacia la superficie es muy rápida y la distribución del organismo se caracteriza por la presencia de un máximo muy marcado cerca de la superficie (Fig. 2).

Además de los movimientos verticales señalados, la observación visual permitió detectar agrupamientos
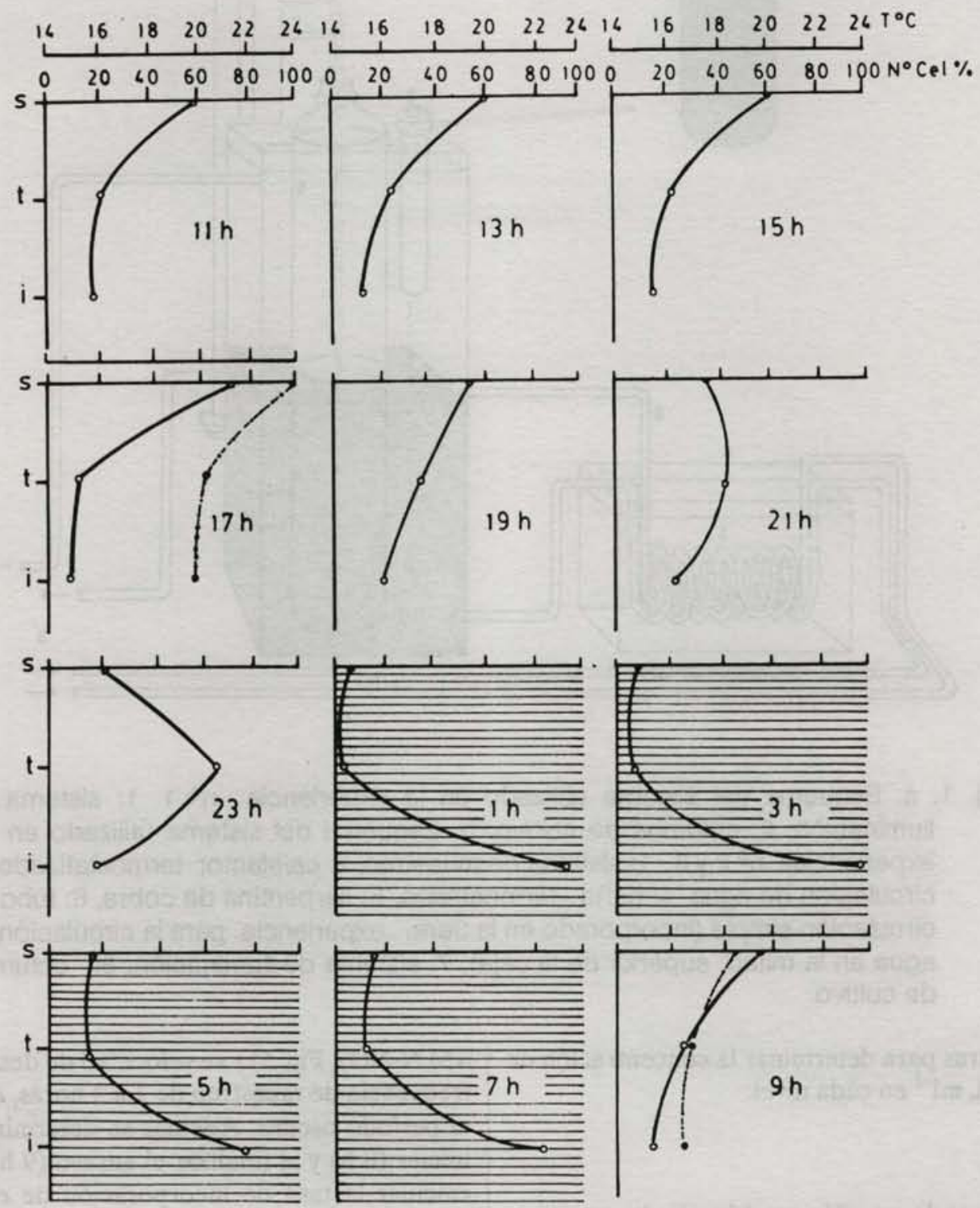

Fig. 2. Distribución vertical de A. excavatum durante la experiencia $\mathrm{n}=1$ (-), y de la temperatura $(-\bullet-)$. Se indica los niveles y horas de extracción de las muestras (s: superior, $t$ : transición, i: inferior). El período luminoso comienza a las $9 \mathrm{~h}$. y finaliza a las $23 \mathrm{~h}$.

período oscuro: $\equiv$ 
celulares característicos, que permiten suponer la existencia de un comportamiento grupal diferenciado para los movimientos de ascenso y descenso de la población. Mientras que el desplazamiento ascensional se realizaba en forma dispersa, el de descenso mostraba agrupamientos celulares en columnas verticales originadas en concentraciones celulares horizontales en forma de bandas paralelas superficiales. El movimiento de estas columnas hacia el fondo presentó una velocidad de desplazamiento elevada, aunque variable, entre 0.35 cm. $\min ^{-1}$ y $4 \mathrm{~cm} \cdot \mathrm{min}^{-1}$.

\section{Experiencia $\mathbf{n}^{\mathbf{2} 2}$}

Aunque la población siguió un comportamiento similar al anteriormente comentado, la presencia de una marcada termoclina produjo un retardo en la iniciación de los movimientos de ascenso y descenso de la población de respuesta al ciclo de luz/oscuridad impuesto al sistema (Fig. 3). Al comienzo del período oscuro, gran parte de la población se mantiene en la superficie, y dos horas después alcanzan a atravesar la termoclina. El mismo retardo se observa en la iniciación del movimiento ascensional, ya que luego de dos horas de iluminación, las células se encuentran concentradas en la zona de la termoclina.

En este ensayo se evidenció el mismo comportamiento grupal en hileras y columnas de descenso notadas en la experiencia anterior.

\section{Experiencia $n^{2} 3$}

Las condiciones requeridas para esta experiencia fueron probadas en la tarde anterior a la jornada programada para el muestreo. Sin embargo en las $15 \mathrm{~h}$ transcurridas desde la adición de nitratos hasta el inicio de
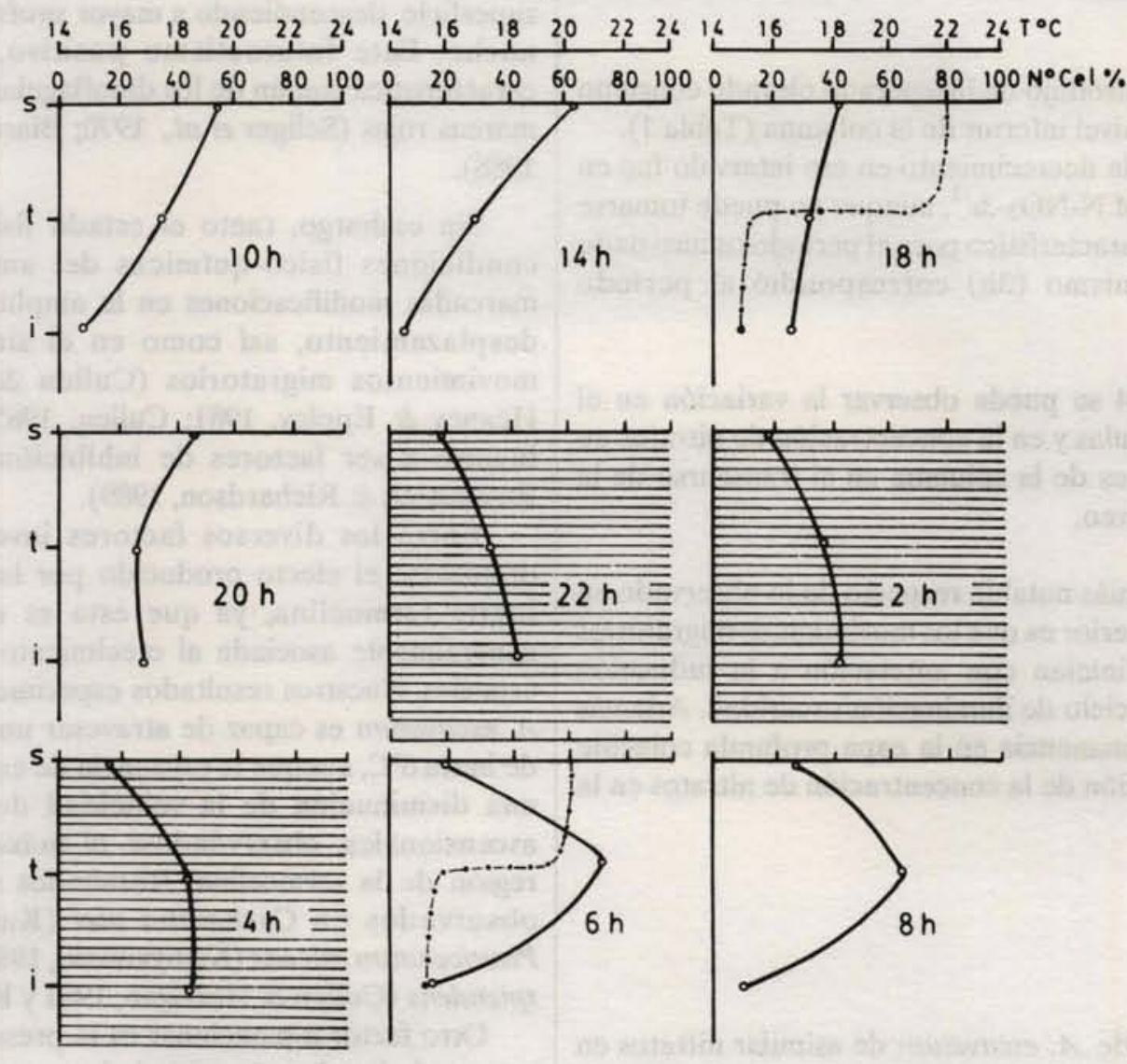

Fig. 3. Distribución vertical de $A$. excavatum durante la experiencia $n^{\circ} 2(-)$, y de

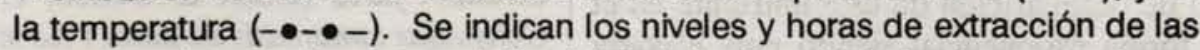
muestras (s: superior, t: transición, i: inferior). El período luminoso comienza a las $6 \mathrm{~h}$ y finaliza a las $20 \mathrm{~h}$.

período oscuro: $\equiv$ 
Tabla 1. Distribución de la concentración de nitratos en la columna experimental (exp. $n=3$ ), a las 18 horas del dia anterior al muestreo y a las 9 horas del mismo.

\begin{tabular}{ccc} 
Fecha & $11-04-83$ & $12-04-83$ \\
Hora & 18.00 & 9.00 \\
& & \\
\hline
\end{tabular}

Concentración

$\left(\mu \mathrm{M} \mathrm{N}-\mathrm{NO}_{3}-\right)$

\begin{tabular}{lll} 
Nivel superior & 0.61 & 0.54 \\
Transición & 0.65 & 0.50 \\
Nivel inferior & 6.11 & 1.48 \\
\hline
\end{tabular}

la experiencia se produjo un inesperado elevado consumo de nitratos en el nivel inferior de la columna (Tabla 1).

La velocidad de decrecimiento en ese intervalo fue en promedio $0.32 \mu \mathrm{M} \mathrm{N}-\mathrm{NO}_{3}-\mathrm{h}^{-1}$, aunque no puede tomarse este valor como característico para el período oscuro dado que parte del mismo (3h) correspondió al período luminoso.

En la Figura 4 se puede observar la variación en el porcentaje de células y en la concentración de nitratos en los distintos niveles de la columna en el transcurso de la jornada de muestreo.

La diferencia más notable respecto de lo observado en la experiencia anterior es que los movimientos migratorios de descenso se inician con antelación a la inducción producida por el ciclo de iluminación/oscuridad. Además el tiempo de permanencia en la capa profunda coincide con una disminución de la concentración de nitratos en la misma.

\section{Experiencia n 4}

La capacidad de $A$. excavatum de asimilar nitratos en la oscuridad fue confirmada en otro ensayo realizado con ese propósito y cuyos resultados se presentan en la Figura 5. La velocidad de desaparición de este nutriente en el medio varió en forma exponencial siendo muy elevada durante la primera hora observándose un valor de $5.29 \mu \mathrm{M}$ $\mathrm{N}-\mathrm{NO}_{3}-\mathrm{h}^{-1}$. En las tres horas subsiguientes disminuyó a
$0.38 \mu \mathrm{M} \mathrm{N}^{-N} \mathrm{~N}_{3}-\mathrm{h}^{-1}$, no registrándose consumo en el resto del período experimental ( 5 horas). Considerando las concentraciones celulares y las variaciones en las concentraciones de nitratos, la incorporación de este nutriente por célula fue de $73.36 \mathrm{pg} \mathrm{N}-\mathrm{NO}_{3}-\mathrm{h}^{-1}$ en la primera hora y en el período subsiguiente fue de $14 \mathrm{pg}$ $\mathrm{N}-\mathrm{NO}_{3}-\cdot \mathrm{cel}^{-1} \cdot \mathrm{h}^{-1}$.

\section{Discusion}

Los estudios experimentales realizados sobre la capacidad de varias especies de dinoflagelados de realizar migraciones verticales, han permitido agruparlas de acuerdo a su patrón de comportamiento (Forward, 1976) en tres tipos principales: 1) Migración hacia la superficie durante el día y descenso o dispersión durante la noche, 2 ) Migración hacia la máxima profundidad durante el día y ascenso hacia la superficie al atardecer, y 3) Migraciones de ascenso y descenso independientes del ciclo de iluminación/oscuridad. Nuestros resultados indican que $A$. excavatum sigue el primer patrón de comportamiento, permaneciendo la mayor parte del período luminoso en la superficie, descendiendo a mayor profundidad durante la noche. Este fototactismo positivo, parece ser una característica común de los dinoflagelados formadores de mareas rojas (Seliger et al., 1970; Blasco, 1978; Ekelund, 1988).

Sin embargo, tanto el estado fisiológico como las condiciones físico-químicas del ambiente producen marcadas modificaciones en la amplitud y velocidad de desplazamiento, así como en el sincronismo de los movimientos migratorios (Cullen \& Horrigan, 1981; Heaney \& Eppley, 1981; Cullen, 1985) pudiendo llegar incluso a ser factores de inhibición (Holligan, 1985; Rasmussen \& Richardson, 1989).

Entre los diversos factores involucrados merece destacarse el efecto producido por la presencia de una fuerte termoclina, ya que esta es una característica generalmente asociada al crecimiento de este grupo de especies. Nuestros resultados experimentales señalan que A. excavatum es capaz de atravesar un gradiente térmico de hasta $6^{\circ} \mathrm{C}$, aunque la existencia de esta barrera produce una disminución de la velocidad de los movimientos ascensionales, observándose el máximo retardo en la región de la termoclina. Resultados similares han sido observados en Cachonina niei (Kamykowski, 1977), Prorocentrum micans (Kamykowski, 1981) y Gymnodinium splendens (Cullen \& Horrigan, 1981 y Kamykowski, 1981).

Otro factor a mencionar es la presencia de haloclinas pronunciadas. La capacidad de cruzar un gradiente de salinidad de $4 \% o \mathrm{~m}^{-1}$ fue confirmada para $P$. micans y Ceratium furca (Edler \& Olsson, 1985) y en el caso de Gonyaulax tamarensis de hasta $1 \%$. $\mathrm{cm}^{-1}$. Para esta última especie el desarrollo de sus poblaciones cerca de la picnoclina es una función de la densidad, la luz y los nutrientes del medio (Rasmussen \& Richardson, 1989). 


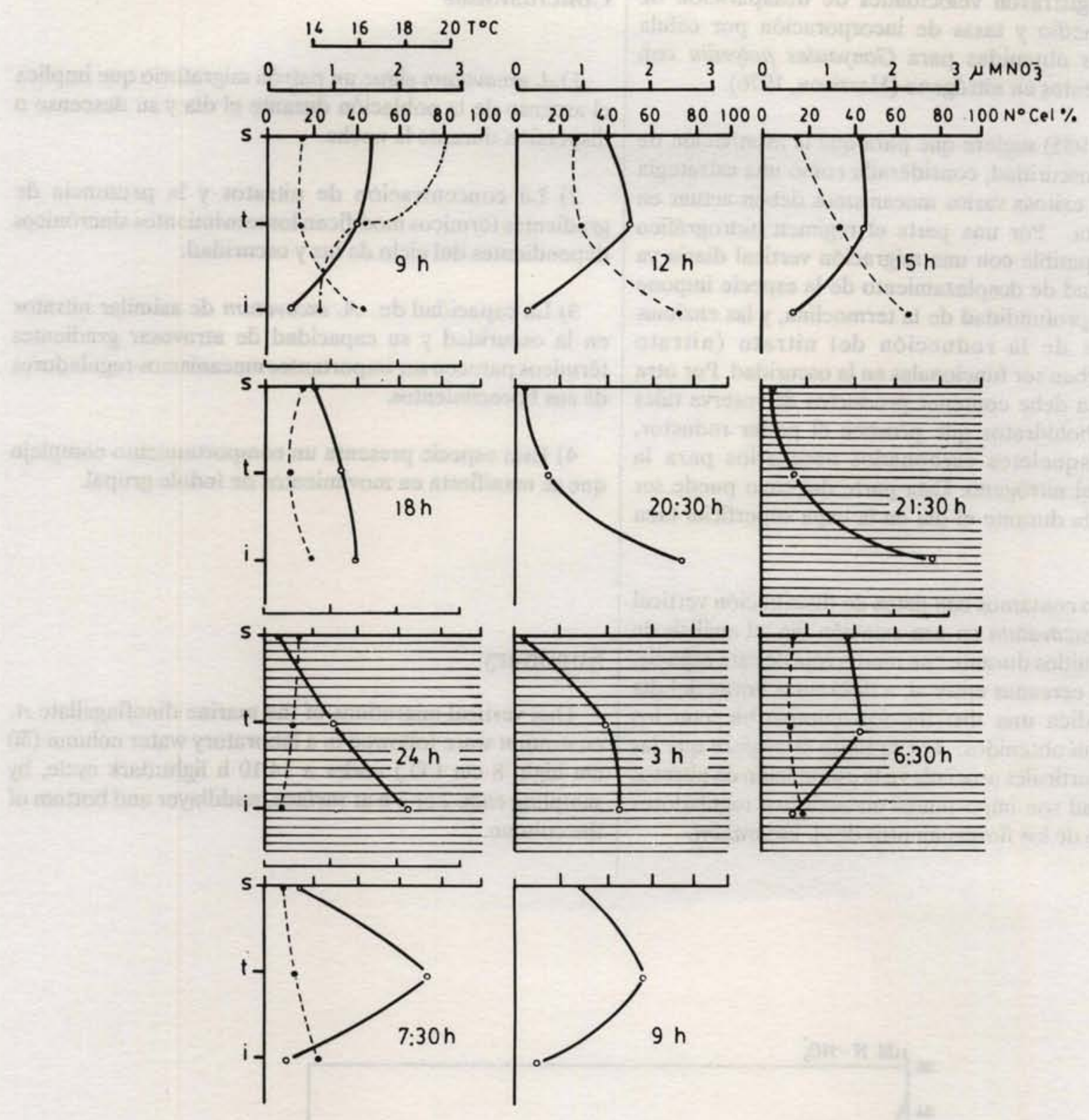

g. 4. Distribución vertical de $A$. excavatum durante la experiencia no3 $(\longrightarrow$, de la temperatura $(-\bullet-\bullet-)$ y de la concentración de nitratos (--). Se inidican los niveles y horas de extracción de las muestras (s:superior, t:transición, i:inferior). El período luminoso comienza a las $7 \mathrm{y}$ finaliza a las $21 \mathrm{~h}$.

Período oscuro: $\equiv$

Nuestros resultados indican también que la existencia de un gradiente en la concentración de nitratos modifica el ritmo migratorio de $A$. excavatum. Los organismos anticipan su descenso unas horas antes de finalizar el período luminoso y ascienden en un período previo al comienzo de éste, alterado su ritmo migratorio. Esta alteración fue observada también para otras especies de dinoflagelados sugiriéndose una relación directa con la disponibilidad de nutrientes en la columna de agua (Cullen \& Horrigan, 1981; Heaney \& Eppley, 1981). Resulta claro, por consiguiente, que el fototactismo no es el único factor de regulación de las migraciones de los dinoflagelados como concluyen Eppley et al. (1968) en base a sus resultados experimentales con Cachonina niei.

$\mathrm{La}$ asimilación de nitratos en la oscuridad, asociada a las migraciones verticales diurnas ha sido considerada una importante ventaja adaptativa de los dinoflagelados (Eppley \& Harrison, 1975; Harrison, 1976). Esta capacidad ha sido comprobada para varias especies en cultivos experimentales (Cullen \& Horrigan, 1981; Heaney \& Eppley, 1981; Dortch \& Maske, 1982; Paasche et al., 1984; Cullen et al., 1985). En las experiencias $n^{2} 3$ y 4 de este 
trabajo, se registraron velocidades de desaparición de nitratos del medio y tasas de incorporación por célula similares a las obtenidas para Gonyaulax polyedra con cultivos deficientes en nitrógeno (Harrison, 1976).

Cullen (1985) sugiere que para que la asimilación de nitratos en la oscuridad, considerada como una estrategia ecológica, sea exitosa varios mecanismos deben actuar en forma conjunta. Por una parte el regimen hidrográfico debe ser compatible con una migración vertical diaria ya que la velocidad de desplazamiento de la especie impone un límite a la profundidad de la termoclina, y las enzimas responsables de la reducción del nitrato (nitrato reductasa), deben ser funcionales en la oscuridad. Por otra parte, la célula debe contener productos de reserva tales como los carbohidratos que proveen el poder reductor, ATP y los esqueletos carbonados necesarios para la asimilación del nitrógeno. Esta parte del ciclo puede ser cumplimentada durante el día en la capa superficial bien iluminada.

Si bien no contamos con datos de distribución vertical diaria de $A$. excavatum en una estación fija, el análisis de los datos obtenidos durante una marea roja de esta especie, en estaciones cercanas entre sí, a diferentes horas del día (Fig. 6), indica una distribución compatible con los resultados aquí obtenidos. Por lo tanto se sugiere que las migraciones verticales asociadas a la asimilación de nitratos en la oscuridad son importantes mecanismos reguladores del desarrollo de los florecimientos de $A$. excavatum.

\section{Conclusiones}

1) A. excavatum sigue un patrón migratorio que implica el ascenso de la población durante el día y su descenso o dispersión durante la noche.

2) La concentración de nitratos y la presencia de gradientes térmicos modifican los movimientos sincrónicos dependientes del ciclo de luz y oscuridad.

3) La capacidad de A. excavatum de asimilar nitratos en la oscuridad y su capacidad de atravesar gradientes térmicos parecen ser importantes mecanismos reguladores de sus florecimientos.

4) Esta especie presenta un comportamiento complejo que se manifiesta en movimientos de índole grupal.

\section{Summary}

Diel vertical migrations of the marine dinoflagellate $A$. excavatum were followed in a laboratory water column ( 50 $\mathrm{cm}$ high, $8 \mathrm{~cm}$ I.D.) under a 14:10 h light:dark cycle, by sampling each 2 or $3 \mathrm{~h}$ at surface, middlayer and bottom of the column.

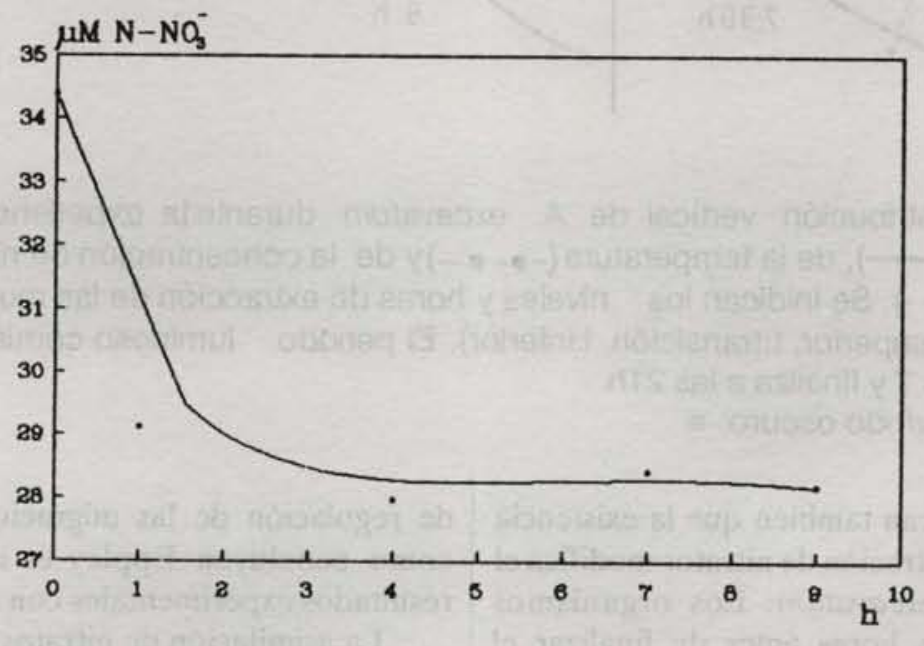

Fig. 5. Resultado de la experiencia $n \div 4$. Asimilación de nitratos en la oscuridad, en un cultivo deficiente en nitrógeno, luego del agregado (tiempo 0) de una solución concentrada de nitrato de potasio. 


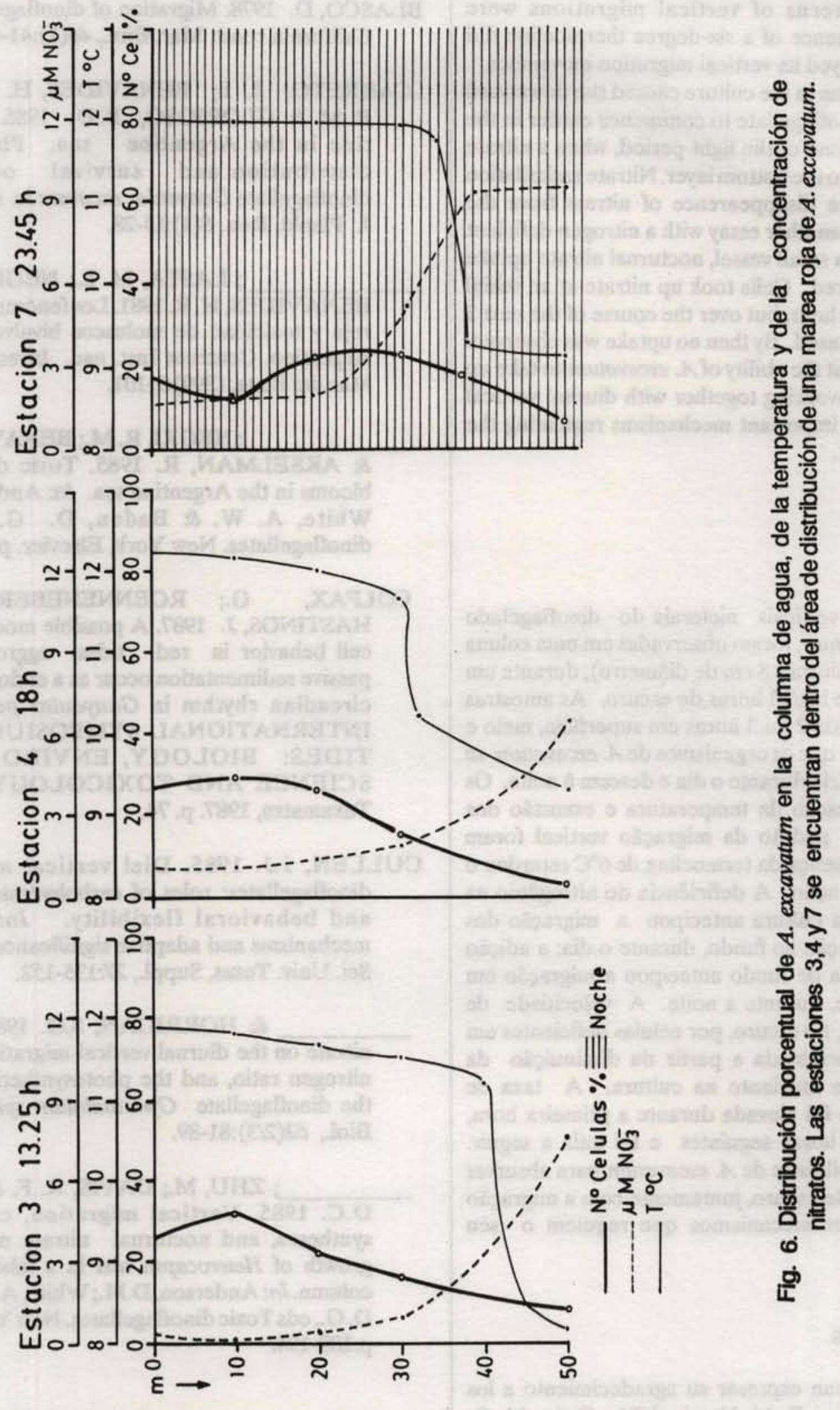


It was found that $A$. excavatum aggregates at surface during daylight and descends at night.

The effects of temperature stratification and nitrogen depletion on patterns of vertical migrations were examined. The presence of a six-degree thermocline did not restrict but delayed its vertical migration movement.

Nitrogen depletion in the culture caused the downward migration of the dinoflagellate to commence earlier in the day and before the end of the light period, when a nitrate solution was added to the bottom layer. Nitrate assimilation was followed by the disappearence of nitrate from the culture medium. In another essay with a nitrogen deficient culture, growing in a small vessel, nocturnal nitrate uptake per cell was measured. Cells took up nitrate at an initial high rate in the first hour, but over the course of the next 3 hours velocity decreased. By then no uptake was observed.

It is suggested that the ability of $A$. excavatum to take up nitrate in the dark working together with diurnal vertical migration could be important mechanisms regulating the bloom development.

\section{Resumo}

As migraçōes verticais nicterais do dinoflagelado Alexandrium excavatum, foram observadas em uma coluna de vidro ( $50 \mathrm{~cm}$ de altura e $8 \mathrm{~cm}$ de diâmetro), durante um ciclo de 14 horas de luz/10 horas de escuro. As amostras foram tomadas a cada 2 ou 3 horas em superfície, meio e fundo. Verificou-se que os organismos de $A$. excavatum se agregam em superfície durante o dia e descem à noite. Os efeitos da estratificaçāo da temperatura e exaustāo dos nutrientes sobre o padrão da migração vertical foram examinados. A presença da termoclina de $6^{\circ} \mathrm{C}$ retardou o movimento de migração. A deficiência do nitrogênio na camada superior da cultura antecipou a migraçāo dos organismos em direção ao fundo, durante o dia; a adição de nitrato à camada de fundo antecipou a migraçāo em direção à superfície, durante a noite. A velocidade de absorçāo do nitrato, no escuro, por células deficientes em nitrogênio, foi determinada a partir da diminuiçāo da concentraçāo desse nutriente na cultura. A taxa de absorção de nitrato foi elevada durante a primeira hora, decresceu nas três horas seguintes e foi nula a seguir. Sugere-se que a habilidade de $A$. excavatum para absorver nitrato no período de escuro, juntamente com a migração vertical, possam ser mecanismos que regulem o seu florescimento.

\section{Agradecimientos}

Los autores desean expresar su agradecimiento a los Lic. H. R. Benavides y R. M. Negri, al Téc. Quím. M. O. Carignan y al Aux. Ing. D. Cuchi Colleoni por la colaboración brindada en el desarrollo del presente trabajo, así como al Prof. Santos Pereyra por la confeccion de las figuras.

\section{Referencias bibliográficas}

BLASCO, D. 1978. Migration of dinoflagellate off Baja California coast. Mar. Biol., 46(1):41-47.

CARRETO, J. I.; BENAVIDES, H. R; NEGRI, R.M. \& GLORIOSO, P.D. 1986. Toxic red tide in the Argentine sea. Phytoplankton distribution and survival of the toxic dinoflagellate Gonyaulax excavata in a frontal area. J. Plankt. Res., 8(1):15-28.

; LASTA, M. L.; NEGRI, R. M. \& BENAVIDES, H. R. 1981. Los fenómenos de marea roja y toxicidad de moluscos bivalvos en el Mar Argentino. Contrnes Inst. nac. Invest. Des. pesq., Mar del Plata, (399):1-101.

;NEGRI, R. M.; BENAVIDES, H. R. \& AKSELMAN, R. 1985. Toxic dinoflagellate blooms in the Argentine sea. In: Anderson, D. M.; White, A. W. \& Baden, D. G., eds Toxic dinoflagellates. New York, Elsevier. p.147-152.

COLFAX, G.; ROENNENEBERG, T. \& HASTINGS, J. 1987. A possible model system for cell behavior in red tides: aggregation and passive sedimentation occur as a endogenous active circadian rhythm in Gonyaulax polyedra. In: INTERNATIONAL SYMPOSIUM ON RED TIDES: BIOLOGY, ENVIRONMENTAL SCIENCE AND TOXICOLOGY. Abstract. Takamatsu, 1987. p. 74.

CULLEN, J.J. 1985. Diel vertical migration by dinoflagellates: roles of carbohydrate metabolism and behavioral flexibility. In: Migration: mechanisms and adaptive significance. Contr. mar. Sci. Univ. Texas, Suppl., 27:135-152.

\& HORRIGAN, S.G. 1981. Effects of nitrate on the diurnal vertical migration, carbon to nitrogen ratio, and the photosynthetic capacity of the dinoflagellate Gymnodinium splendens. Mar. Biol., 62(2/3):81-89.

; ZHU, M.; DAVIS, R. F. \& PIERSON, D.C. 1985. Vertical migration, carbohydrate syntheses, and nocturnal nitrate uptake during growth of Heterocapsa niei in a laboratory water column. In: Anderson, D.M.; White, A. W. \& Baden, D. G., eds Toxic dinoflagellates. New York, Elsevier. p.189-194.

DORTCH, Q. \& MASKE, H. 1982. Dark uptake of nitrate and nitrate reductase activity of a red-tide population off Peru. Mar. Ecol.-Prog. Ser., 9(3):299-303. 
EDLER, L. \& OLSSON, P. 1985. Observations on diel migration of Ceratium furca and Prorocentrum micans in a stratified bay on the Swedish west coast. In: Anderson, D. M.; White, A. W. \& Baden, D. G., eds Toxic dinoflagellates. New York, Elsevier. p. 195-200.

EKELUND, N. G. A. 1988. Photomovements in Gyrodinium dorsum, Gyrodinium aureolum and Chlamydomonas reinhardtii. Doctoral dissertation. University of Lund. Sweeden.

EPPLEY, R. W. \& HARRISON, S. G. 1975. Physiological ecology of Gonyaulax polyedra a red tide dinoflagellate of southern California. In: Lo Cicero, V. R., ed. Toxic dinoflagellate blooms. Massachusetts, Science and Technology Foundation. p.11-22.

; HOLM-HANSEN, O. \& STRICKLAND, J. D. 1968. Some observations on the vertical migration of dinoflagellates. J. Phycol., 4:333-340.

FORWARD, R.B. 1976. Light and diurnal vertical migration: photobehavior and photophysiology of plankton. Photochem. Photobiol. Rev., 1:157-209.

FOURNIER, R. O. 1978. Membrane filters for estimating cell numbers. In: Sournia, A., ed. Phytoplankton manual. Monogr. oceanogr. Methodol., U.N., (6):108-112.

GUILLARD, R. L. \& RYTHER, J. H. 1962. Studies on marine planktonic diatoms. I. Cyclotella nana Husted and Detonula confervacea (Cleve) Gran. Can. J. Microbiol., 8:229-239.

HARRISON, W. G. 1976. Nitrate metabolism of the red tide dinoflagellate Gonyaulax excavata Stein. J. expl. mar. Biol. Ecol., 21(3):199-209.

HEANEY, S. I. \& EPPLEY, R. W. 1981. Light, temperature and nitrogen as interacting factors affecting diel vertical migration of dinoflagellates in culture. J. Plankt. Res., 2(2):331-344.
HOLLIGAN, P. M. 1985. Marine dinoflagellate blooms. Growth strategies and environmental explotation. In: Anderson, D. M.; White, A. W. \& Baden, D. G., eds Toxic dinoflagellates. New York, Elsevier. p.133-139.

HORSTMANN, V. 1980. Observations on the peculiar diurnal migration of red tide Dinophyceae in tropical shallow waters. J. Phycol., 16:481-485.

KAMYKOWSKI, D. 1981. Laboratory experiments on the diurnal migration of motile phytoplankton through temperature gradients. Mar. Biol., 62(1):57-64.

\& ZENTARA, S. J. 1977. The diurnal vertical migration of phytoplankton through temperature gradients. Limnol. Oceanogr., 22(1):148-151.

PAASCHE, E.; BRYCESON, I. \& TANGEN, K. 1984. Interespecific variation in dark nitrogen uptake by dinoflagellates. J. Phycol., 20:394-401.

RASMUSSEN, J. \& RICHARDSON, K. 1989. Response of Gonyaulax excavata to the presence of a pycnocline in an artificial water column. J. Plankt. Res., 11(4):747-762.

SELIGER, H.; CARPENTER, J.; LOFTUS, M. \& ELROY, W. 1970. Mechanisms for the accumulation of high concentration of dinoflagellates in a bioluminiscent bay. Limnol. Oceanogr., 15 (2):234-245.

UTERMÖHL, H. 1958. Zur Vervolkomnug der quantitativen Phytoplankton-Methodik. Mitt. int. Verein. theor. angew. Limnol., $9(1): 1-38$.

WOOD, E.; ARMSTRONG, P. \& RICHARDS, R. A. 1967. Determination of nitrate in seawater by cadmium-copper reduction to nitrite. J.mar. biol. Ass. U.K., 47(1):23-31.

(Manuscrito recibido 31 Enero 1991; revisado 5 Febrero 1992; aprobado 7 Agosto 1992) 